\title{
Development of $3 \times 3$ DOF blocking structural elements to enhance the computational intensity of iterative linear solver
}

\author{
Naoki MORITA*, Kazuo YONEKURA ${ }^{* *}$, Ichiro YASUZUMI ${ }^{* *}$, Mitsuyoshi TSUNORI ${ }^{* *}$, \\ Gaku HASHIMOTO* and Hiroshi OKUDA* \\ * Graduate School of Frontier Sciences, The University of Tokyo \\ 5-1-5 Kashiwanoha, Kashiwa, Chiba, 277-8563, Japan \\ E-mail:morita@multi.k.u-tokyo.ac.jp \\ ** IHI Corporation \\ 1, Shin-nakahara-cho, Isogo-ku, Yokohama, Kanagawa, 235-8501, Japan
}

Received 8 February 2016

\begin{abstract}
Structural elements which have 6 degrees of freedom (DOFs) at each node, such as shell and beam elements, are widely used in structural analysis. A matrix originating from finite element method (FEM) is stored in some sparse matrix storage format with a suitable block size for the number of DOFs at each node. When both 6 DOF structural elements and general 3 DOF solid elements are employed, the sparse matrix storage format becomes complicated due to combination of different block sizes. High computational efficiency of finite element analysis has become more important in large-scale structural problems. The complicated storage format leads to deterioration of computing performance in solving linear equations by an iterative procedure, conjugate gradient (CG) iterations for example. A computational technique is required that allows us to use existing parallel linear solvers without deteriorating the performance for solving linear equation systems originating from combination of solid and structural elements. This research aims to develop $3 \times 3$ DOF blocking structural elements to enhance the computational intensity of iterative linear solver, such as the CG method. As numerical results, the proposed $3 \times 3$ DOF blocking elements have shown better performance for each CG iteration than the conventional structural elements. The computational efficiencies are $95.0 \%$ with single thread execution and $76.6 \%$ with 8 -thread execution of a theoretical peak performance based on the STREAM benchmark.
\end{abstract}

Key words : Finite element method, Structural element, Shell element, Beam element, Sparse matrix storage format, Conjugate gradient method, Computational intensity

\section{Introduction}

Large-scale finite element analysis has become more important in various engineering and industrial fields to obtain numerical results with high accuracy. Structural elements, such as shell and beam elements, are mainly employed for modeling complicate structural components in further detail. Solid elements have 3 degrees of freedom (DOFs) at each node in three-dimensional analysis. Beam and shell elements have 6 DOFs: the sum of translational 3 DOFs and rotational 3 DOFs, for example, Bernoulli-Euler beam element (Han, 1999) and MITC4 shell element (Dvorkin and Bathe, 1984) with a drilling DOF (Hughes and Brezzi, 1989, Pimpinelli, 2004 and Long et al., 2006). Structural elements generally lead to good numerical results for industrial structures using the much smaller number of nodes than solid elements. In addition, this is an advantage in terms of memory usage in large-scale computation.

Currently, a matrix originating from finete element method (FEM) is stored in some sparse matrix storage format with a suitable block size for the number of DOFs at each node. However, when linear equation systems originating from combination of structural and solid elements are solved, the sparse matrix storage format has become complicated due to combination of different block sizes. General parallel linear solvers are not specialized in such complicated storage 


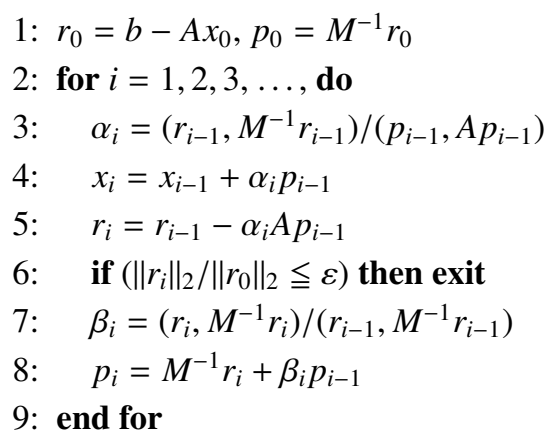

Fig. 1 Preconditioned CG method where $r$ is the residual vector, $p$ is the search direction vector, $M$ is the preconditioner, the subscript $i$ is the iteration index, $\varepsilon$ is the convergence tolerance for solving linear systems $A x=b$

format to solve various linear equations. Furthermore, solver performance is one of the most important issues in largescale analysis. It is desired to show good computing performance even though solid elements and shell or beam elements are included in a model. An easier way of solving linear equation systems originating from combination of different block sizes is to transform a $6 \times 6$ block submatrix to $3 \times 3$ block submatrices.

In this paper, we propose $3 \times 3$ DOF blocking structural elements to enhance the computational intensity of iterative linear solver, such as the conjugate gradient (CG) method. A matrix originating from the proposed elements is stored in a sparse matrix storage format with $3 \times 3$ block submatrices. The elements proposed here allow us to use existing parallel linear solvers without deteriorating the computing performance. Compared with $6 \times 6$ DOF blocking, $3 \times 3$ DOF blocking gives better performance to solve linear equations on super computers.

This paper is structured as follows: in section 2, we state an overview of finite element method and data storage format. In section 3, we present $3 \times 3$ DOF blocking structural elements to enhance the computational intensity. In section 4, we show numerical experiments and performance of SpMV kernel in the CG method with the proposed elements. In section 5 , we present some concluding remarks.

\section{Outline of finite element method, data storage format and SpMV kernel}

\subsection{Finite element method}

This research focuses on small deformation problems. FEM is a method for discretizing partial differential equations. In finite element analysis (FEA), symmetric positive definite linear systems are solved as

$$
A x=b
$$

where $A$ is the sparse stiffness matrix, $x$ is the displacement vector and $b$ is the external load vector.

In this paper, considering memory requirement and parallel performance for solving a huge matrix originating from large-scale FEA, linear systems are solved by the preconditioned CG method that is a well known iterative solver to solve positive define symmetric linear systems (Saad, 2003). Figure 1 shows an algorithm of the preconditioned CG method for solving $A x=b$. This method requires a large amount of sparse matrix-vector multiplication (SpMV) and solution time is dominated by the SpMV computation.

\section{2. $3 \times 3$ block compressed sparse row storage format}

Compressed sparse row (CSR) storage format is one of the current standard storage formats of sparse matrices in FEA. Block compressed sparse row (BCSR) storage format is extended as a blocking type of CSR. Non-zero pattern of sparse matrices originating from solid elements in three-dimensional FEA can be represented as succinct pattern with $3 \times 3$ block submatrices.

Figure 2 shows a schematic representation of $3 \times 3$ BCSR. In this figure, each non-zero value is stored in non-zero pattern of a matrix which consists of $3 \times 3$ block submatrices. Compared to CSR, BCSR is more efficient because indices of each column and positions of each non-zero value are concerned only with submatrices.

\subsection{SpMV kernel}

SpMV is an important operation and widely used in many applications. Especially, SpMV dominates execution time for each iteration in iterative linear solvers, such as the CG method. Thus performance of the CG method considerably depends on performance of SpMV kernel. 


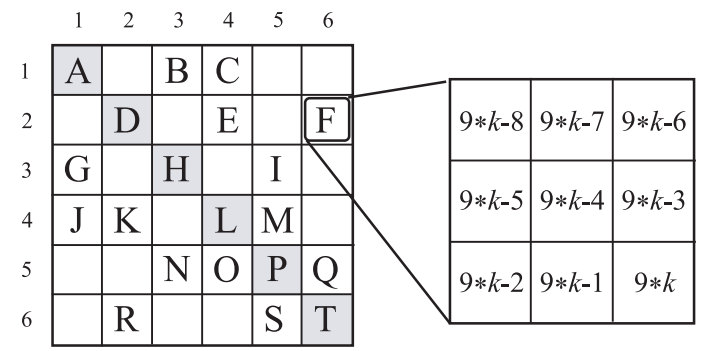

\author{
value $=[\mathrm{A}, \mathrm{B}, \mathrm{C}, \mathrm{D}, \mathrm{E}, \mathrm{F}, \mathrm{G}, \mathrm{H}, \mathrm{I}, \mathrm{J}, \mathrm{K}$, \\ L, M, N, O, P, Q, R, S, T] \\ index $=[0,3,6,9,13,17,20]$ \\ item $=[1,3,4,2,4,6,1,3,5,1,2$, \\ $4,5,3,4,5,6,2,5,6]$
}

Fig. 2 Schematic representation of $3 \times 3$ BCSR storage format, where the real data array value holds non-zero value of submatrices, the integer array index shows indeces of each column, the integer array item shows row positions of each non-zero value and $k$ is the temporary integer data.

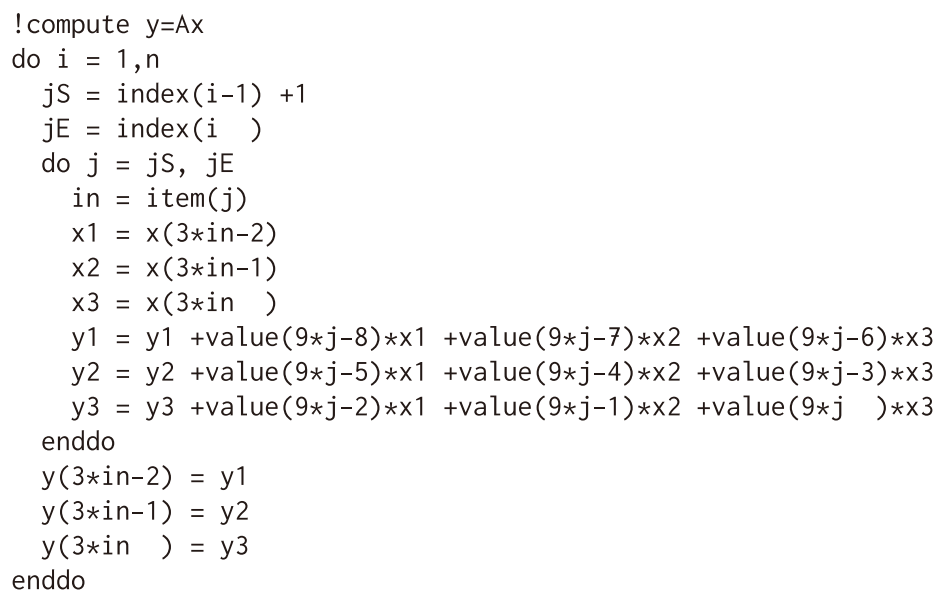

Fig. 3 Fortran90 program of SpMV kernel for $y=A x$ in $3 \times 3$ BCSR storage format, where value is the matrix nonzero value, $x$ is the right-hand side vector, $y$ is the left-hand side vector, inde $x$ is the index array, item is the item array, $n$ is the martix size, $i, j, j S$ and $j E$ are the temporary integer data, and $x 1, x 2, x 3, y 1, y 2$ and $y 3$ are the temporary real data.

Figure 3 shows Fortran90 program of SpMV kernel in $3 \times 3$ BCSR storage format. B/F (Byte per FLOPS) rate of this $3 \times 3$ block SpMV kernel is calculated following the procedure below. In the kernel, assuming that $x, x 1, x 2, x 3, y, y 1, y 2$ and $y 3$ are on cache, 9 double-precision real data Value and a single-precision integer data Item must be loaded for each inner loop. The total amount of the read data is 76 Bytes (the sum of 9 variables Value $\times 8$ Bytes and 1 variable Item $\times$ 4 Bytes). The total number of operation is 18 FLOPS (the sum of 9 add and 9 multiply operations) for each inner loop $j$. Therefore B/F rate of the SpMV kernel is 4.22 (= 76 Bytes / 18 FLOPS). In the same way, B/F rate of the $6 \times 6$ block SpMV kernel is 4.05 (= 292 Bytes / 72 FLOPS). Based on the B/F rates of the SpMV kernels, the $6 \times 6$ block strategy provides better performance than the $3 \times 3$ block strategy. However, actual performance of SpMV is influenced by cache miss.

\title{
3. 3×3 DOF Blocking Structural Elements
}

In this section, we propose $3 \times 3$ DOF blocking structural elements. The proposed structural elements utilize data structures of solid elements in an FEM program that has been already implemented. In this study, the proposed beam elements, triangular shell elements and quadrilateral shell elements have data structures of 4-node tetrahedron elements, 6-node prism elements and the 8-node hexahedral elements, respectively. Figure 4 shows the conventional structural elements, i.e. the 2-node beam element, the 3-node triangular shell element and the 4-node quadrilateral shell element. The total number of DOFs of the beam element is 12 , that of the triangular shell element is 18 , and that of the quadrilateral shell element is 24 . Figure 5 shows the proposed $3 \times 3$ blocking structural elements: 4-node beam element with 2 dummy nodes, 6-node triangular shell element with 3 dummy nodes and 8-node quadrilateral shell element with 4 dummy nodes. The total number of DOFs in the proposed element is same as that in the conventional element. To compute the stiffness matrix and external vector for the proposed element, the components of the matrix and vector for the conventional structural element are permutated.

Figure 6 shows a schematic representation of the conventional 3-node triangular shell element. Figure 7 shows a 


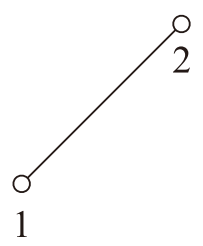

(a) 2-node beam element

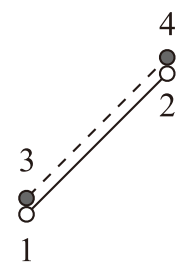

(d) 4-node beam element with 2 dummy nodes

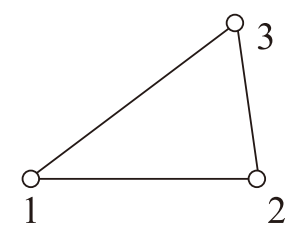

(b) 3-node triangular shell element

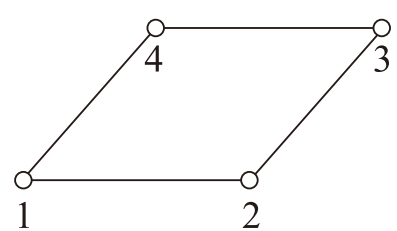

(c) 4-node quadrilateral shell element

Fig. 4 The conventional structural elements

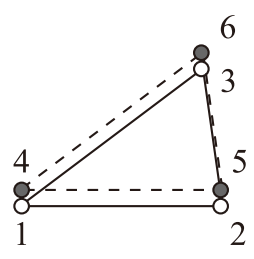

(e) 6-node triangular shell element with 3 dummy nodes

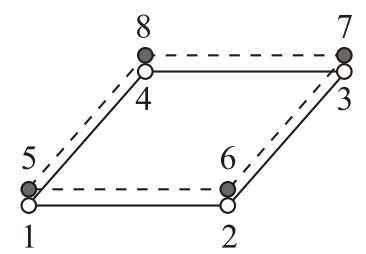

(f) 8-node quadrilateral shell element with 4 dummy nodes

Fig. $53 \times 3$ DOF blocking structural elements

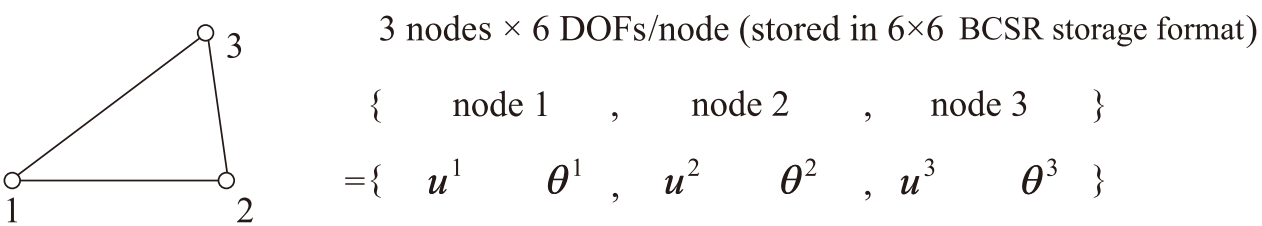

Fig. 6 3-node triangular shell element where $u^{i}$ is the translational DOFs, $\theta^{i}$ is the rotational DOFs and the superscript $i(i=1,2,3)$ is the local node number

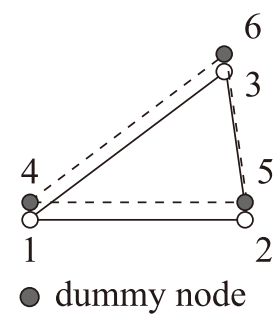

6 nodes $\times 3$ DOFs $/$ node (stored in $3 \times 3$ BCSR storage format)

\{ node 1 , node 2 , node 3 , node 4 , node 5 , node 6 \}

$=\left\{u^{1}, u^{2}, u^{3}, \theta^{4}, \theta^{5}, \theta^{6}\right\}$

Fig. 7 6-node triangular shell element where $u^{i}$ is the translational DOFs at each node, $\theta^{i}$ is the rotational DOFs and the superscript $i(i=1,2,3,4,5,6)$ is the local node number

schematic representation of 6-node triangular shell element with 3 dummy nodes. The conventional 3-node triangular shell element has 6DOFs: translational 3 DOFs and rotational 3 DOFs at each node. By contrast, the proposed element has $3 \mathrm{DOF}$ at each node and translational DOFs and rotational DOFs are stored separately. The translational DOFs are defined at the former 3 nodes and the rotational DOFs are defined at the later (dummy) 3 nodes. The coordinates of the dummy node and connectivity between the dummy nodes are not necessary for computation of geometric information. In Fig.7, geometric information, such as covariant vectors, contravariant vectors and Jacobian, is computed from three nodes 1,2 and 3 and the connectivity 1-2-3.

The proposed elements are implemented in FrontISTR, the open-source parallel FEM program based on domain decomposition method (Okuda, 2013). Implementation of the proposed elements utilizes the existing element definitions for the 4-node tetrahedral element, 6-node prism solid and 8-node hexahedral solid element in FrontISTR. In a setup phase of FEA with FrontISTR, dummy nodes are generated automatically.

\section{Numerical experiments}

\subsection{Numerical environment and conditions}

In this section, we report numerical results of SpMV in the CG solver on the K-computer. The system configuration of the K-computer is shown in Table 1 . The K-computer system is the massively parallel super computer which consists of 82,944 nodes $(663,552$ cores) and the peak performance is $10.62 \mathrm{Pflops}$. Each node has 8 cores $(2.0 \mathrm{GHz} / \mathrm{core})$ and 
Table 1 Specification of the K-computer

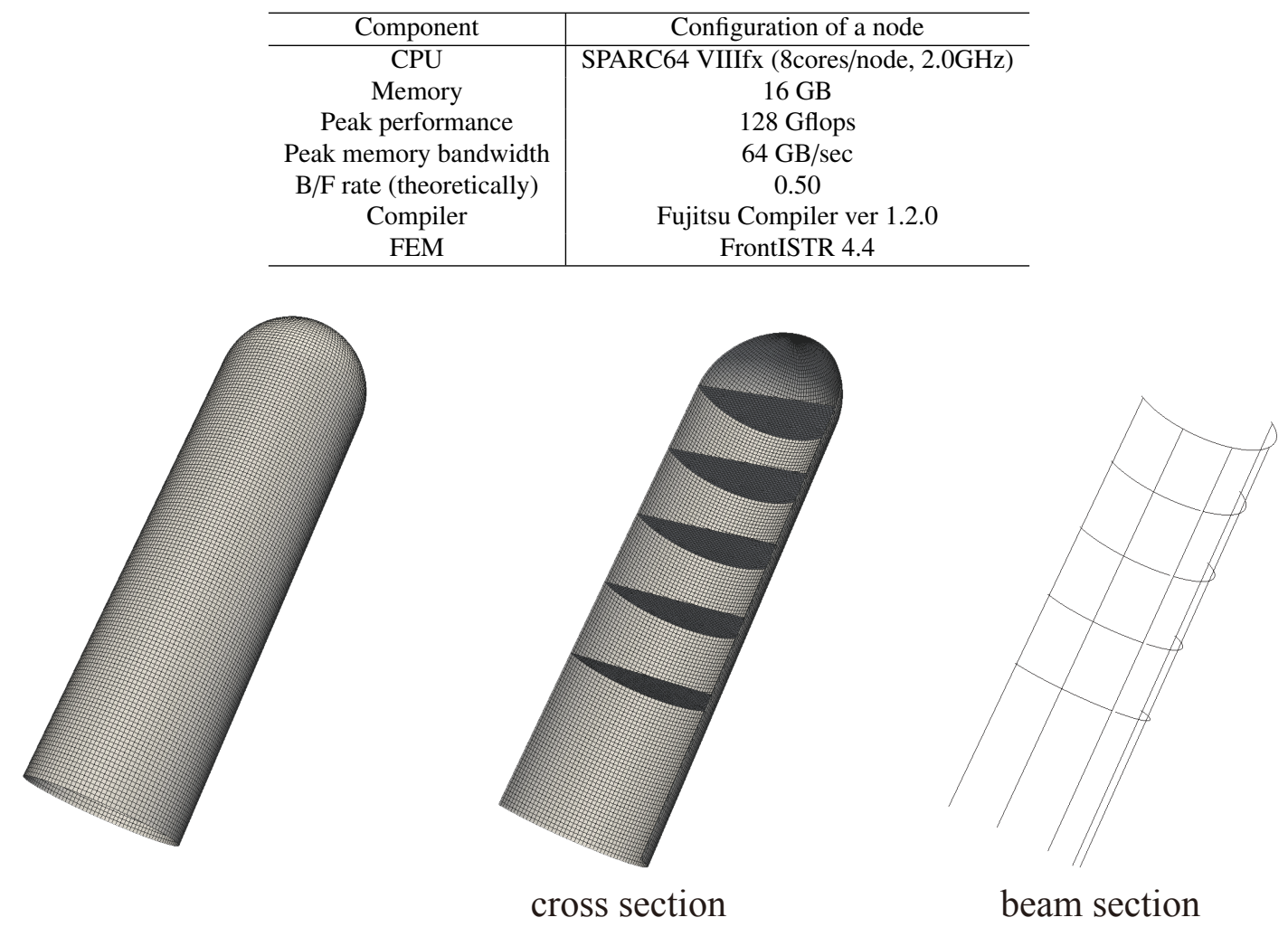

Fig. 8 Analysis mesh of a typical cylindrical column which has MITC4 shell elements and 2-node BernoulliEuler beam elements

the peak performance of each node is 128 Gflops. Peak memory bandwidth of each node is $64 \mathrm{~GB} / \mathrm{s}$ and thus B/F rate is 0.5 . The STREAM benchmark memory performance of each node is $36 \mathrm{~GB} / \mathrm{s}$ and thus B/F rate is 0.28 (Ono et al., 2014). Thereby theoretical performance of the $3 \times 3$ DOF blocking SpMV kernel is $6.63 \%(=0.28 / 4.22)$ and theoretical performance of the $6 \times 6$ DOF blocking SpMV kernel is $6.91 \%(=0.28 / 4.05)$ based on the STREAM benchmark.

The performances of the presented and conventional methods are measured with the Fujitsu profiler on the Kcomputer.

\subsection{Numerical example}

In this example, we solve a matrix originating from a cylindrical column shaped model of MITC4 shell element (Dvorkin and Bathe, 1984) and Bernoulli-Euler beam element (Han, 1999) as shown in Fig. 8. The open line in the mesh is completely fixed and the whole model is loaded with the volume force (gravity) along a longitudinal direction. The analysis mesh has 24,590 nodes, 31,180 elements (29,620 shell elements and 1,560 beam elements), 147,540 DOFs, $8,010,936$ non-zero entries and sparsity is $2.73 \%$. The computation is executed following 2 patterns; single OpenMP thread of serial computation, and 8 OpenMP threads of parallel computation. The purpose of this example is to evaluate the performance of the proposed $3 \times 3$ DOF blocking and conventional $6 \times 6$ DOF blocking elements in the SpMV computation. The performance of the SpMV computation does not depend on the number of DOFs but the cache hit ratio.

Table 2 shows numerical results of SpMV kernel performance in the CG solver. On SpMV multiplication with single thread execution, the conventional $6 \times 6$ DOF blocking elements perform at $4.97 \%$ and the proposed $3 \times 3$ DOF blocking elements perform at $6.30 \%$ of the theoretical perk performance of the CPU. The computational efficiency of the proposed elements is $95.0 \%$ of the STREAM benchmark peak performance (6.63\%). On SpMV multiplication with 8-OpenMPthreads execution, the conventional $6 \times 6$ DOF blocking elements perform at $4.25 \%$ and the proposed $3 \times 3$ DOF blocking elements perform at $5.08 \%$ of the theoretical perk performance. The computational efficiency of the proposed elements is $76.6 \%$ of the STREAM peak performance $(6.63 \%)$. 
Table 2 Numerical results of SpMV kernel performance in the CG solver where "Num. of cores" is the number of cores, "Storage format" is the employed storage format, "Performance [Mflops]" is the performance of SpMV kernel for each CG iteration, "Performance/peak [\%]" is the percentage of peak performance, "Mem. throughput $[\mathrm{GB} / \mathrm{s}]$ " is the memory throughput of SpMV kernel for each CG iteration, "Mem. throughput/peak [\%]" is the percentage of peak memory throughput.

\begin{tabular}{c|c|rr|rr}
\hline \hline Num. of cores & Storage format & \multicolumn{1}{|c|}{$\begin{array}{c}\text { Performance } \\
\text { [Mflops] }\end{array}$} & $\begin{array}{c}\text { Performance } \\
\text { /Peak [\%] }\end{array}$ & $\begin{array}{c}\text { Mem. Throughput } \\
\text { [GB/sec] }\end{array}$ & $\begin{array}{c}\text { Mem. throughput } \\
\text { /Peak [\%] }\end{array}$ \\
\hline \multirow{2}{*}{1} & $3 \times 3$ blocking & $1,007.19$ & 6.30 & 4.74 & 7.41 \\
& $6 \times 6$ blocking & 795.39 & 4.97 & 3.48 & 5.44 \\
\hline \multirow{2}{*}{8} & $3 \times 3$ blocking & $6,499.30$ & 5.08 & 29.72 & 46.44 \\
& $6 \times 6$ blocking & $5,440.40$ & 4.25 & 23.18 & 36.22 \\
\hline
\end{tabular}

\section{Conclusion}

In this paper, $3 \times 3$ DOF blocking structural elements was developed to enhance the computational intensity of iterative linear solver. The conclusions are as follows;

- The proposed method allows us to use the existing parallel linear solvers without deteriorating the computing performance for solving linear equation systems originating from combination of different block sizes using both structural and solid elements.

- As numerical results, the proposed $3 \times 3$ DOF blocking elements showed better performance for CG iterations than the $6 \times 6$ DOF blocking elements. The performance of the proposed method is at most $6.30 \%$ of the theoretical peak performance of a computing node on the K-computer. The computational efficiencies of the SpMV kernel with single thread execution and 8-thread execution are $95.0 \%$ and $76.6 \%$ of the theoretical peak performance respectively.

\section{Acknowledgements}

Part of the results is obtained by using the K computer at the RIKEN Advanced Institute for Computational Science (Proposal number hp130091).

\section{References}

Dvorkin, E. N. and Bathe, K. J., A Continuum Mechanics Based Four-node Shell Element for General Non-linear Analysis, Engineering Computations, Vol. 1 (1984), pp.77-88.

Han, M. S., Benaroya, H. and Wei, T., Dynamics of transversely vibrating beams using four engineering theories, Journal of Sound and Vibration, Vol. 255, No. 5 (1999), pp.935-988.

Hughes, T. and Brezzi, F., On drilling degrees of freedom, Computer Methods in Applied Mechanics and Engineering, Vol. 72, No. 1 (1989), pp.105-121.

Long, S., Geyer, S. and Groenwold, A., A numerical study of the effect of penalty parameters for membrane elements with independent rotation fields and penalized equilibrium, Finite Elements in Analysis and Design, Vol. 42, No. 8 (2006), pp.757-765.

Okuda, H., Nonlinear Structual Analysis Open Software FrontISTR, available from 〈http://www.ciss.iss.u-tokyo.ac.jp/ riss/english/project/structure/FISTR_JE_1303.pdf), (accessed on 15 February, 2016).

Ono, K., Chiba, S., Inoue, S., and Minami, K., Performance Improvement of Iterative Methods using a Bit-Representation Technique for Coefficient Matrices, Vecpar 2014, Vol. 15 (2014).

Pimpinelli, G., An assumed strain quadrilateral element with drilling degrees of freedom, Finite Elements in Analysis and Design, Vol. 41, No. 3 (2004), pp.267-283.

STREAM: Sustainable Memory Bandwidth in High Performance Computers, available from /http://www.cs.virginia.edu/ stream $\rangle$, (accessed on 15 February, 2016).

Saad, Y., Iterative Methods for Sparse Linear Systems, 2nd Edition (2003), Society for Industrial and Applied Mathematics. 